\title{
A comparison of the quality assurance of four dosimetric tools for intensity modulated radiation therapy
}

\author{
Jaeman Son ${ }^{1,2}$, Taesung Baek ${ }^{1,4}$, Boram Lee ${ }^{1,5}$, Dongho Shin², Sung Yong Park \\ Jeonghoon Park², Young Kyung Lim², Se Byeong Lee², Jooyoung Kim², Myonggeun Yoon
}

\author{
${ }^{1}$ Department of Bio-Convergence Engineering, Korea University, Seoul, Korea \\ 2 Proton Therapy Center, National Cancer Center, Goyang, Korea \\ ${ }^{3}$ McLaren Proton Therapy Center, Karmanos Cancer Institute, Flint, MI, USA \\ ${ }^{4}$ Department of Radiation Oncology, Ilsan Hospital, Goyang, Korea \\ ${ }^{5}$ Department of Radiation Oncology, Sun Hospital, Daejeon, Korea
}

Radiol Oncol 2015; 49(3): 307-313.

Received 10 November 2014

Accepted 18 January 2015

Correspondence to: Myonggeun Yoon, Department of Bio-convergence Engineering, Korea University, Anam Ro 145, Seongbuk-gu, Seoul 136-701, Korea. Phone: +82 23290 5651; Fax: +82 2940 2829; E-mail: radioyoon@korea.ac.kr

Disclosure: The authors declare no conflict of interest.

Background. This study was designed to compare the quality assurance (QA) results of four dosimetric tools used for intensity modulated radiation therapy (IMRT) and to suggest universal criteria for the passing rate in QA, irrespective of the dosimetric tool used.

Materials and methods. Thirty fields of IMRT plans from five patients were selected, followed by irradiation onto radiochromic film, a diode array (Mapcheck), an ion chamber array (MatriXX) and an electronic portal imaging device (EPID) for patient-specific QA. The measured doses from the four dosimetric tools were compared with the dose calculated by the treatment planning system. The passing rates of the four dosimetric tools were calculated using the gamma index method, using as criteria a dose difference of $3 \%$ and a distance-to-agreement of $3 \mathrm{~mm}$.

Results. The QA results based on Mapcheck, MatriXX and EPID showed good agreement, with average passing rates of $99.61 \%, 99.04 \%$ and $99.29 \%$, respectively. However, the average passing rate based on film measurement was significantly lower, $95.88 \%$. The average uncertainty ( 1 standard deviation) of passing rates for 6 intensity modulated fields was around 0.31 for film measurement, larger than those of the other three dosimetric tools.

Conclusions. QA results and consistencies depend on the choice of dosimetric tool. Universal passing rates should depend on the normalization or inter-comparisons of dosimetric tools if more than one dosimetric tool is used for patient specific QA.

Key words: intensity modulated radiation therapy; quality assurance; dosimetric tool; gamma index

\section{Introduction}

Radiation therapy is one of the most widely used cancer treatment methods. Among the methods used are $3 \mathrm{D}$ conformal radiation therapy $(3 \mathrm{D}$ CRT), intensity modulated radiotherapy (IMRT), and particle beam therapy. IMRT uses a multi-leaf collimator (MLC) to vary the intensity of the beam delivered to the tumour. ${ }^{1}$ Since IMRT requires fine control of the MLC during irradiation, cau- tion must be exercised in delivering radiation. ${ }^{2-4}$ Treatment quality assurance (QA) is therefore necessary to determine the difference between calculated and actual dose distributions., , $^{5}$

QA for conventional IMRT treatment is widely performed using an ion chamber and film, with the ion chamber used to measure absolute dose at each location and film used for 2D relative dose comparisons..$^{7-9}$ Although film has the great advantage of high resolution, it has several disadvantages, 
including the need to change film for every beam test and its dependence on beam energy, processing conditions, and external light. Recently developed radiation therapy methods have greater accuracy, with new dosimetry tools developed to overcome the disadvantages of film measurements. Among these tools are a diode detectorbased 2D diode array dosimeter ${ }^{10}$ (MapCheck2; Sun Nuclear Corporation, Melbourne, Florida), a 2D ion chamber array dosimeter ${ }^{11}$ (I'mRT MatriXX; IBA) and portal dosimetry using an electronic portal imaging device ${ }^{12}$ (EPID). The gamma evaluation method is generally used to verify the actual dose distribution that will be delivered to the patient during IMRT. ${ }^{13}$ This method is based on a comparison of the calculated 2D dose map from treatment planning system (TPS) with the measured 2D dose map from each dosimetric tool. Although there is no general consensus, QA results are considered acceptable when the passing rate is greater than $95 \%$ using as criteria a tolerance of dose difference (DD) of $3 \%$ and a tolerance for distance to agreement (DTA) of $3 \mathrm{~mm} \cdot{ }^{14}$

Table 1 shows an example of QA results using film, Mapcheck, MatriXX and portal dosimetry based on gamma evaluation for 10 randomly selected treatment plans undergoing IMRT at four different institutions in Korea. Each of 4 hospitals sorted out 10 IMRT QA results using one of four dosimetric tools and analysed the passing rates depending on the QA tools. Therefore, this data shows the general passing rates of IMRT QA depending on the dosimetric tools. The mean \pm standard deviation (SD) passing rates $(\gamma \% \leq 1)$ for film, Mapcheck, MatriXX and EPID were $96.80 \pm 0.94 \%$, $98.90 \pm 0.55 \%, 99.40 \pm 0.48 \%$ and $97.10 \pm 1.12 \%$, respectively. Thus, passing rates are dependent on the dosimetric tool used, with mean passing rates being lowest for film and highest for MatriXX. This example suggests that passing rates of $95 \%$ for film measurement and portal dosimetry are not equivalent. ${ }^{15,16}$ Many institutions, however, have not set an acceptance level for each tool but have universally set $95 \%$ as the acceptance level for all tools. The current emphasis on treatment QA for patients suggests the need for specific guidelines for each specific dosimetric tool. Although guidelines have been proposed ${ }^{17}$, they were only for acceptable doses and allowable errors for each body part, and did not include passing rates for different dosimetric tools.

In this study, four different dosimetric tools were used for quality assurance method of IMRT plans from five patients and their patient spe-
TABLE 1. Average passing rates for film, diode array (Mapcheck), ion chamber array (MatriXX) and electronic portal imaging device (EPID) for intensity modulated radiation therapy (IMRT) quality assurance (QA) in four different institutions in Korea

\begin{tabular}{|c|c|c|c|c|}
\hline & Film & Mapcheck & MatriXX & EPID \\
\hline Mean & 96.80 & 98.90 & 99.40 & 97.10 \\
\hline $\begin{array}{l}\text { Standard } \\
\text { deviation }\end{array}$ & 0.94 & 0.55 & 0.48 & 1.12 \\
\hline
\end{tabular}

cific QA results were compared. The correlations among these dosimetric tools were used to determine reasonable tolerance levels for each.

\section{Materials and methods}

The IMRT plans for five patients undergoing radiation therapy, modelled using the Eclipse treatment planning system Ver. 8.9. (Varian Medical Systems, Salt Lake City, UT, USA) with anisotropic analytical algorithm (AAA), were used for this study. ${ }^{18}$ Figure 1 shows the experimental setup of IMRT QA with film, Mapcheck, MatriXX and EPID. The gamma index method was used to compare the TPS dose with measured doses, using dose difference (DD) criteria of 3\% and distance-to-agreement criteria (DTA) of $3 \mathrm{~mm}$. The reference dose map was the calculated dose maps from TPS in our analysis. The gamma index value was calculated at all 2-dimensional points, with the percentage of points with a gamma index value $\leq 1$ and meeting the DD and DTA criteria being the passing rate. The passing rates of the four dosimetric tools were compared.

\section{Film QA}

The traditional method of QA for IMRT is 2-dimensional testing using film. We used commercial Gafchromic EBT2 film (International Specialty Products, New Jersey, USA ${ }^{19}$ and an Epson Expression 10000-XL flatbed film scanner (Epson, California, USA), with a resolution of $0.38 \times 0.38$ $\mathrm{mm}^{2}$. Film was calibrated using an ion chamber, with more calibration points used for low dose areas to enhance accuracy. Doses were measured at a source to axis distance (SAD) of $100 \mathrm{~cm}$, with film located at a depth of $10 \mathrm{~cm}$ of solid slab phantom and a gantry angle of $0^{\circ}$. Radiological Imaging Technology IMRT software (Ver. 5.2, Colorado Springs, CO, USA) was used to verify dose delivery after the beam measurement. 


\section{MapCheck QA}

MapCheck2 (Sun Nuclear; Melbourne, FL, USA) is a relatively new dosimetric tool, consisting of diode detectors in a 2D array and a field size of $32 \mathrm{~cm}$ x $26 \mathrm{~cm}$. The matrix is composed of 1,527 diodes, spaced $7.07 \mathrm{~mm}$ apart, with each having an active detector area of $0.64 \mathrm{~mm}^{2}$ and the entire matrix having active detector size of $24.4 \times 24.4 \mathrm{~cm}^{2} .^{20}$ Similar to film QA, dose was measured at $8 \mathrm{~cm}$ depth of Mapcheck dedicated phantom, Mapphan (Sun Nuclear, Melbourne, FL, USA) at a gantry angle of $0^{\circ}$. Mapcheck dedicated software (MapCHECK2, Ver 5.01.05, Sun Nuclear, Melbourne, FL, USA), was used to verify the dose delivery after the beam measurement.

\section{MatriXX QA}

The MatriXX (IBA Dosimetry GmbH, Schwarzenbruck, Germany) is similar to Mapcheck but has ionization chambers rather than diode detectors in a 2D array. ${ }^{21,22}$ Although MatriXX has fewer ion chambers than Mapcheck has diode detectors, the ion chambers yield more stable data than the diodes. $^{23}$ The 1,024 ionization chambers of MatriXX are aligned in a parallel pattern in a $32 \times 32$ grid, with the diameter, height, volume and detector spacing of each ion chamber being $4.5 \mathrm{~mm}, 7.62$ $\mathrm{mm}, 0.08 \mathrm{cc}$ and $7.62 \mathrm{~mm}$, respectively. The gantry angle was $0^{\circ}$ and the beam was investigated using a $5 \mathrm{~cm}$ solid water phantom on top of the MatriXX. OmniPro-I'mRT (Ver 1.7.0021, IBA Dosimetry, Germany), a MatriXX-dedicated software program, was used to verify dose delivery after beam irradiation.

\section{EPID QA (portal dosimetry)}

We used an aSi-based EPID (aS500, Varian Medical Systems, Palo Alto, CA) attached to a Varian Clinac iX Linear accelerator (Varian Medical Systems, Palo Alto, CA, USA). ${ }^{12}$ This EPID has a resolution of $0.784 \times 0.784 \mathrm{~mm}^{2}$ with an array of 512 x 384 pixels, thus having higher resolution than Mapcheck or MatriXX, and a field size of 40 $x 30 \mathrm{~cm}^{2}$. The source to axis distance (SAD) was set at $100 \mathrm{~cm}$ and the beam was investigated at a gantry angle of $0^{\circ}$. Measurement with EPID was measured with no phantom and EPID dedicated software (Eclipse, Ver 8.9, Varian Medical System, USA) was used to verify dose delivery after the beam measurement.

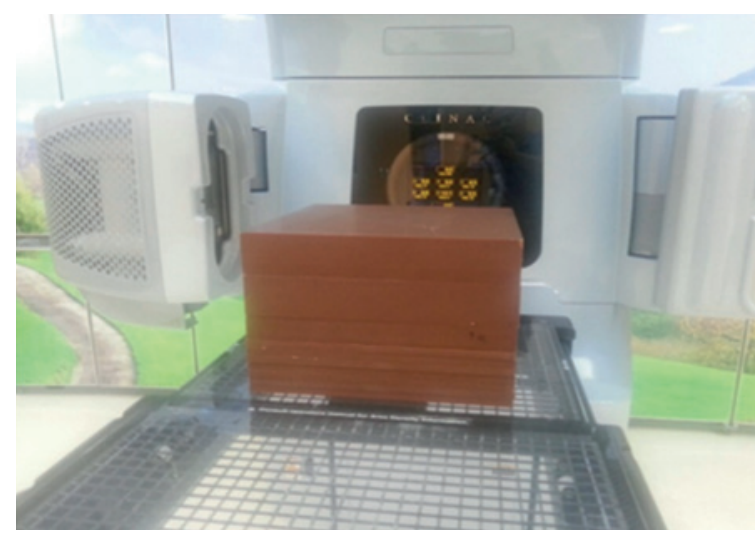

(A)

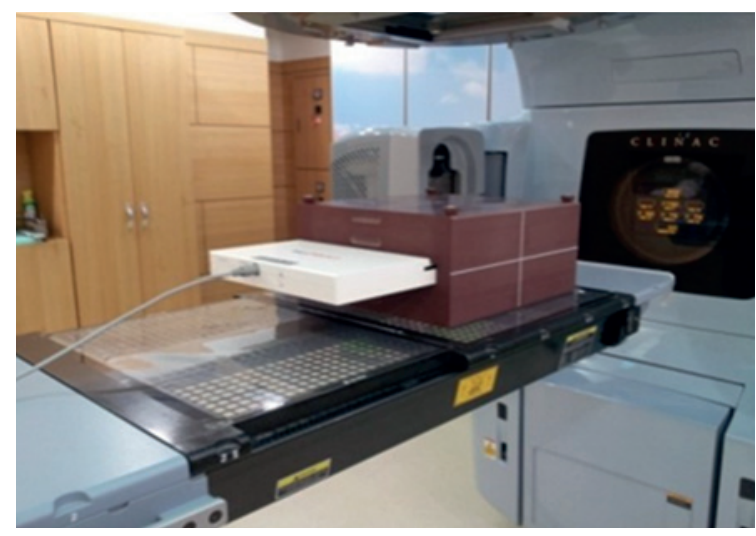

(B)

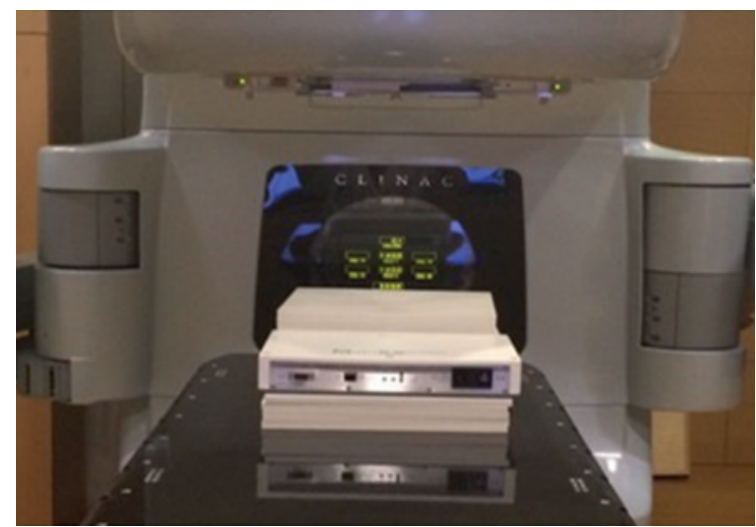

(C)

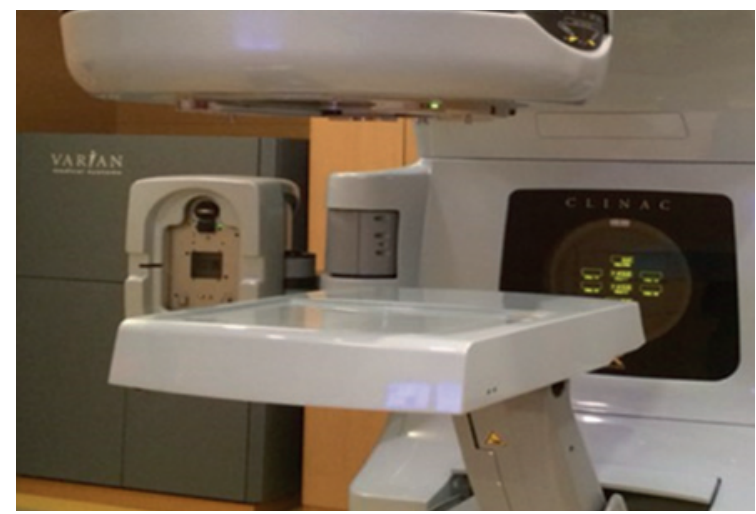

FIGURE 1. Pictures of the experimental setup for IMRT QA using various dosimetric tools. (A) Film, (B) Mapcheck, (C) ion chamber array (MatriXX), (D) Portal dosimetry. 
TABLE 2. Mean passing rates based on the gamma index method for the treatment fields of each patient using film, diode array (Mapcheck), ion chamber array (MatriXX), and electronic portal imaging device (EPID)

\begin{tabular}{lcccccc}
\hline & Patient 1 & Patient 2 & Patient 3 & Patient 4 & Patient 5 \\
\hline Film & 97.42 & 95.42 & 95.83 & 94.80 & 95.92 & 95.88 \\
Mapcheck & 100.00 & 99.45 & 100.00 & 98.90 & 99.70 & 99.61 \\
MatriXX & 99.10 & 99.26 & 98.84 & 98.82 & 99.20 & 99.04 \\
EPID & 99.42 & 99.12 & 99.20 & 99.52 & 99.20 & 99.29 \\
\hline
\end{tabular}

\section{Results}

Doses calculated using TPS were compared with doses measured by the four dosimetric tools based on gamma evaluation $(3 \% / 3 \mathrm{~mm}$, threshold $15 \%)$. Figure 2 shows examples of gamma evaluation results using film, Mapcheck, MatriXX and EPID for IMRT QA. Although Table 1 shows the general passing rates of IMRT QA depending on the dosimetric tools, the result can be dependent on the patient selected in each institution. To clarify the dependency of QA result on dosimetric tools, one should carry out IMRT QA of same patient using different dosimetric tools. Table 2 shows the mean passing rates, based on the gamma index method, for the treatment fields of each patient using film,
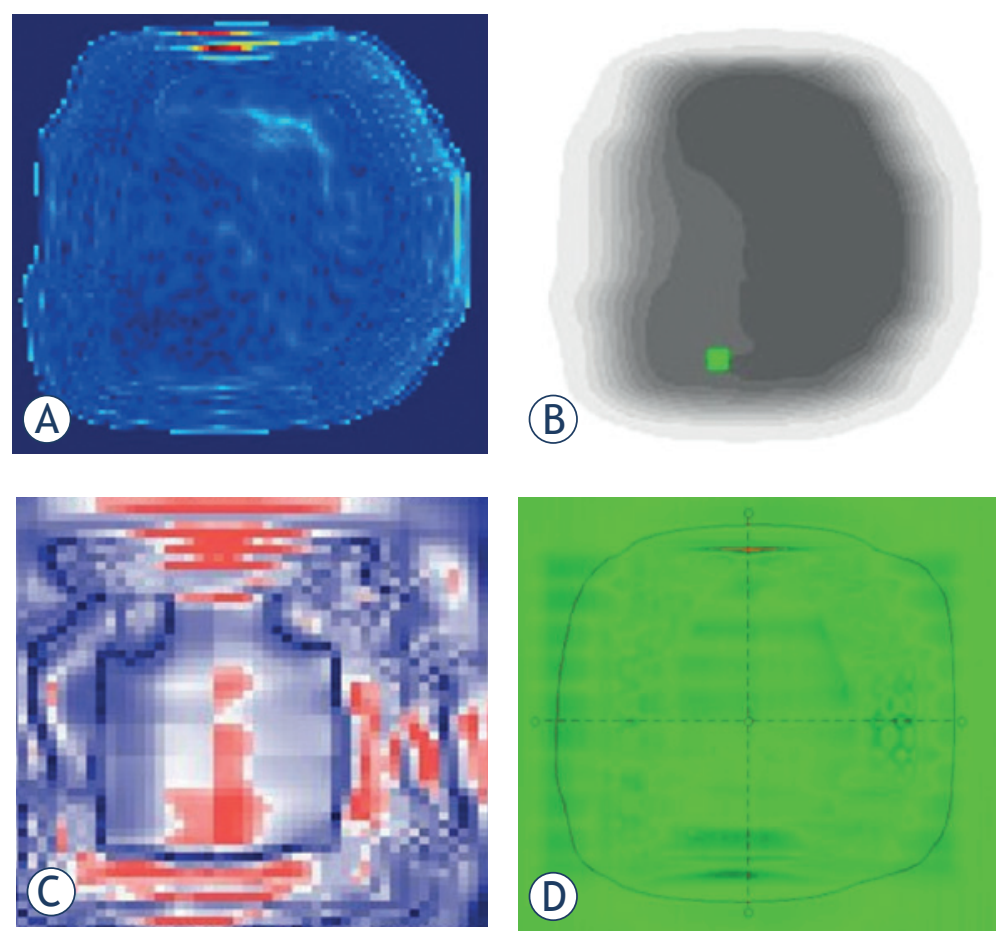

FIGURE 2. 2D images of the passing rate based on gamma evaluation for various dosimetric tools. (A) Film, (B) diode array (Mapcheck), (C) ion chamber array (MatriXX), (D) Portal dosimetry.
Mapcheck, MatriXX, and EPID. The values measured with the four dosimetry tools showed good agreement with the calculated values for all five patients. The mean \pm standard deviation (SD) passing rates $(\gamma \% \leq 1)$ for film, Mapcheck, MatriXX and EPID for 30 IMRT fields of five patients were 95.88 $\pm 0.87 \%, 99.61 \pm 0.41 \%, 99.04 \pm 0.18 \%$ and $99.29 \pm$ $0.15 \%$, respectively. Although all four dosimetry tools met the acceptable passing rate of $95 \%$, these tools showed some differences in measuring the same beam, with the gamma index being much lower for film than for the three other tools.

To show fluctuations for each dosimetric tool, we assessed the passing rates of three consecutive measurement results using film, Mapcheck, MatriXX and EPID based on gamma index values for 6 fields of patient 1 from Table 2. This means that QA was carried out 3 times, repetitively. As shown in Table 3, the gamma index results were similar, regardless of the number of measurements, for Mapcheck, MatriXX and EPID. Film, however, showed higher a standard deviation (i.e., fluctuation) for three consecutive measurements than for the other dosimetirc tools. For example, the fluctuation of film measurement for field 1 was 0.59 which is much higher than $0.00,0.00,0.05$ for Mapcheck, MatriXX and EPID, respectively.

\section{Discussion}

This comparison of gamma indices for EBT film, Mapcheck, MatriXX, and EPID showed differences in dose distribution when using these various dosimetric tools to carry out the quality assurance for the same patients undergoing IMRT. Even with using the same dosimetry tool, the results differed slightly for each measurement of the same field.

The passing rates based on film measurement were much lower than those using the three other dosimetric tools (Table 2). To check the uncertainty of film for the exact same beam, QA was carried out only one time but 3 films were inserted in the 
TABLE 3. Passing rates of three consecutive measurement results using film, diode array (Mapcheck), ion chamber array (MatriXX) and electronic portal imaging device (EPID) based on gamma index values for 6 fields of patient 1 . The data shown for patient 1 in Table 2 is the average of first measurement set of data in Table 3

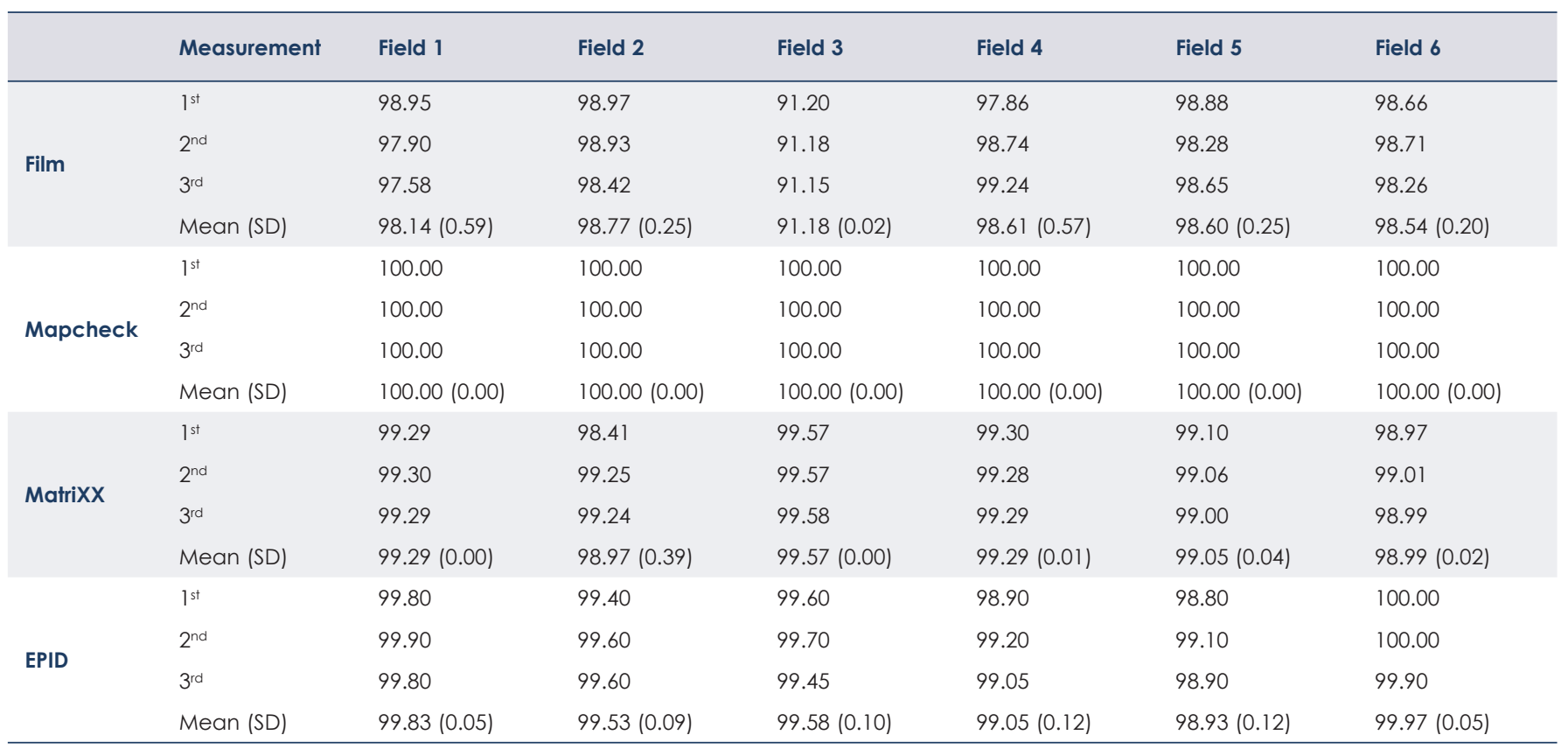

SD = standard deviation

phantom. To irradiate the exactly same beam, one should remove the unstable low dose part of the beam caused by scattering, leakage, etc. To do that, we did carry out QA not with individual field but with composite fields, which may remove the low dose part in the analysis. Assessment of the passing rates based on gamma evaluation showed uncertainties ranging from 0.11 to 0.39 (Table 4). Despite these uncertainties, however, the results were reasonably stable, suggesting that a single measurement would be sufficient for QA.

In general, uncertainties tend to be higher for low dose film measurements. ${ }^{20}$ We therefore investigated a beam with a universally-tripled-beam intensity (i.e., tripled monitor unit) on each field to confirm the decrease of gamma index with low dose. Table 5 shows the passing rate using film measurements for 3-fold increased beam intensity for 6 fields of patient 1,2,4. Compared with the results shown in Table 3 , the mean passing rate increased, indicating that, in general, high and stable gamma index results with film can be obtained at higher beam intensity (i.e., increased monitor units), comparable to the results from the three other dosimetric tools. Table 5 also shows that standard deviation is decreased with increased beam intensity. Therefore, QA using film should be
TABLE 4. Passing rates based on gamma evaluation using 3 films in the same location for intensity modulated radiation therapy (IMRT) quality assurance (QA) of each patient

\begin{tabular}{lccccc}
\hline & Patient 1 & Patient 2 & Patient 3 & Patient 4 & Patient 5 \\
\hline \multirow{3}{*}{ Film } & 98.36 & 95.29 & 98.51 & 98.08 & 99.41 \\
& 97.47 & 95.47 & 98.55 & 98.19 & 99.14 \\
\multirow{2}{*}{ Mean } & 97.63 & 95.65 & 98.87 & 98.36 & 99.27 \\
SD & 97.82 & 95.47 & 98.64 & 98.21 & 99.27 \\
\hline
\end{tabular}

performed at a sufficiently high intensity beam to reduce the uncertainties from low doses. Although raising the threshold level will lead to clearing of low dose, it may not appropriate since it can cause only the high dose area to be set as region of interest. If one wants to use normal treatment MU in QA, careful calibration of film should be used to decrease the standard deviation of film for low dose area.

While the QA results based on Mapcheck, MatriXX and EPID showed good agreement among themselves by showing average passing rates of $99.61 \%, 99.04 \%$ and $99.29 \%$, respectively, the aver- 
TABLE 5. Passing rates of film measurements based on gamma evaluation for 3-fold increased beam intensity (i.e., monitor unit) for 6 fields of patient 1

\begin{tabular}{lllllllll}
\hline & Field1 & Field2 & Field3 & Field4 & Field5 & Field6 & Mean & SD \\
\hline Patient1 & 99.23 & 99.45 & 99.20 & 99.09 & 99.04 & 99.21 & 99.20 \\
Patient2 & 98.63 & 98.04 & 98.03 & 97.91 & 96.80 & 97.15 & 97.76 \\
Patient4 & 98.77 & 98.67 & 99.23 & 99.40 & 99.93 & 98.30 & 9.13 & 9.05 \\
\hline
\end{tabular}

age passing rate based on film measurement was significantly lower, $95.88 \%$. The QA result seems to depend on the unique characteristics of each dosimetric tool such as depth of measurement, its resolution, etc. These experimental results suggest that the criteria of the passing rate based on film measurement should be decreased about 3\% compared to the criteria for other dosimetric tools. If one wants to use universal criteria for the passing rate for all four dosimetric tools, one should increase the beam intensity about three times if the film is used as a measurement tool. Further study is needed to suggest the more reliable universal passing rate with large number of case studies.

\section{Conclusions}

We have used film, Mapcheck, MatriXX and EPID to evaluate their dosimetric properties for IMRT QA. While the measured dose maps with Mapcheck, MatriXX and EPID agreed well with the calculated dose maps of TPS, the passing rate was noticeably lower for film measurements. It seems that different passing rates in QA results may partially stem from the different resolution (or measured dose matrix) of four dosimetric tools. Use of film for IMRT QA should be implemented using beams of sufficiently high intensity to be compatible with the results of Mapcheck, MatriXX, and EPID. Our results suggest that setting an acceptance level based on the correlation of various dosimetric tools is a more correct method than simply setting the same acceptance level for all of these tools.

\section{Acknowledgment}

This work was supported by the Nuclear Safety Research Program through the Korea Radiation Safety Foundation (KORSAFe) and the Nuclear Safety and Security Commission(NSSC), Republic of Korea (Grant No. 1305033) and by the National Nuclear R\&D Program through the National
Research Foundation of Korea (NRF), funded by the Ministry of Education, Science and Technology (NRF-2013M2A2A7067089), and by Basic Science Research Program through the National Research Foundation of Korea (NRF) funded by the Ministry of Education, Science and Technology (NRF2013R1A1A2007630).

\section{References}

1. Lee $N$, Terezakis S. Intensity-modulated radiation therapy. J Surg Oncol 2008; 97: 691-6.

2. Langer $M$, Brown R, Urie M, Leong J, Stracher M, Shapiro J. Large scale optimization of beam weights under dose-volume restrictions. Int J Radiat Oncol Biol Phys 1990; 18: 887-93.

3. Morrill SM, Lane RG, Wong JA, Rosen II. Dose-volume considerations with linear programming optimization. Med Phys 1991; 18: 1201-10.

4. Langer M, Leong J. Optimization of beam weights under dose-volume restrictions. Int J Radiat Oncol Biol Phys 1987; 13: 1255-60.

5. Van Esch A, Bohsung J, Sorvari P, Tenhunen M, Paiusco M, lori M, et al. Acceptance tests and quality control $(\mathrm{OC})$ procedures for the clinical implementation of intensity modulated radiotherapy (IMRT) using inverse planning and the sliding window technique: experience from five radiotherapy departments. Radiother Oncol 2002; 65: 53-70.

6. Palta JR, Liu C, Li JG. Quality assurance of intensity-modulated radiation therapy. Int J Radiat Oncol, Biol, Phys 2008; 71: S108-12.

7. Tsai Ph D J-S, Wazer MD DE, Ling MD MN, Wu MD JK, Fagundes MD M DiPetrillo MD T, et al. Dosimetric verification of the dynamic intensitymodulated radiation therapy of 92 patients. Int J Radiat Oncol Biol Phys 1998; 40: 1213-30.

8. Fraass B, Doppke K, Hunt M, Kutcher G, Starkschall G, Stern R, et al. American Association of Physicists in Medicine Radiation Therapy Committee Task Group 53: quality assurance for clinical radiotherapy treatment planning. Med Phys 1998; 25: 1773-829.

9. Ezzell GA, Galvin JM, Low D, Palta JR, Rosen I, Sharpe MB, et al. Guidance document on delivery, treatment planning, and clinical implementation of IMRT: Report of the IMRT subcommittee of the AAPM radiation therapy committee. Med Phys 2003; 30: 2089.

10. Jursinic PA, Nelms BE. A 2-D diode array and analysis software for verification of intensity modulated radiation therapy delivery. Med Phys 2003; 30: 870-9.

11. Poppe B, Blechschmidt A, Djouguela A, Kollhoff R, Rubach A, Willborn KC, et al. Two-dimensional ionization chamber arrays for IMRT plan verification. Med Phys 2006; 33: 1005-15.

12. Van Esch A, Depuydt T, Huyskens DP. The use of an aSi-based EPID for routine absolute dosimetric pre-treatment verification of dynamic IMRT fields. Radiother Oncol 2004; 71: 223-34.

13. Low DA, Harms WB, Mutic S, Purdy JA. A technique for the quantitative evaluation of dose distributions. Med Phys 1998; 25: 656-61.

14. Howell RM, Smith I, Jarrio CS. Establishing action levels for EPID-based QA for IMRT. J Appl Clin Med Phys 2008; 9: 16-25. 
15. Ezzell GA, Burmeister JW, Dogan N, LoSasso TJ, Mechalakos JG, Mihailidis $D$, et al. IMRT commissioning: Multiple institution planning and dosimetry comparisons, a report from AAPM Task Group 119. Med Phys 2009; 36: 5359.

16. Basran PS, Woo MK. An analysis of tolerance levels in IMRT quality assurance procedures. Med Phys 2008; 35: 2300-7.

17. Nelms BE, Simon JA. A survey on IMRT QA analysis. J Appl Clin Med Phys 2007; 8: 2448 .

18. Bragg CM, Wingate K, Conway J. Clinical implications of the anisotropic analytical algorithm for IMRT treatment planning and verification. Radiother Oncol 2008; 86: 276-84

19. Zeidan OA, Stephenson SA, Meeks SL, Wagner TH, Willoughby TR, Kupelian $\mathrm{PA}$, et al. Characterization and use of EBT radiochromic film for IMRT dose verification. Med Phys 2006; 33: 4064-72.

20. Letourneau D, Gulam M, Yan D, Oldham M, Wong JW. Evaluation of a 2D diode array for IMRT quality assurance. Radiother Oncol 2004; 70: 199-206.

21. Saminathan S, Manickam R, Chandraraj V, Supe SS. Dosimetric study of 2D ion chamber array matrix for the modern radiotherapy treatment verification. J Appl Clin Med Phys 2010; 11: 3076.

22. Herzen J, Todorovic M, Cremers F, Platz V, Albers D, Bartels A, et al. Dosimetric evaluation of a 2D pixel ionization chamber for implementation in clinical routine. Phys Med Biol 2007; 52: 1197-208.

23. Li JG, Yan G, Liu C. Comparison of two commercial detector arrays for IMRT quality assurance. J Appl Clin Med Phys 2009; 10: 2942. 